\title{
Developing an acute oncology support smartphone app for Wales
}

\author{
Authors: Kate Davies ${ }^{\mathrm{A}}$, Abigail Hayward, ${ }^{\mathrm{B}}$ Jenna Chapman, ${ }^{\mathrm{C}}$ Rosie Roberts, ${ }^{\mathrm{B}, \mathrm{C}}$ Paul Edmonds ${ }^{\mathrm{C}}$ and Hilary Williams ${ }^{\mathrm{C}}$
}

\section{Aims}

We developed an Android/iPhone app hoping to increase junior doctors' confidence and competence in managing cancer emergencies by consolidating Welsh acute oncology guidelines in an interactive, free to download format.

\section{Methods}

A small multidisciplinary cross-site working group was established in 2015. Current local oncology guidelines were extensively reviewed and edited with the aim of making them concise, user friendly and unambiguous. Other medical apps were reviewed to inform ideas about style and useful content. Across Wales, agreement to protocols was obtained and funding was then secured from the Velindre Cancer Centre Fundraising charity. A digital marketing agency was appointed. Possible formats were considered in meetings with them and then guidelines were ordered into the agreed 'Recognition, Assessment, Response' framework. Some ancillary checklists were added as were useful contact details to make the content as useful as possible. A prototype app was then produced and tested. A video advertising the app was developed, describing the app as being 'like having your own personal oncologist providing you with expert advice exactly when you need it'. The final app was released at a launch event at Velindre Cancer Centre, extensively publicised on social media, in October 2017.

\section{Results}

Prior to the launch of the app we surveyed junior doctor opinion around management of acute oncological emergencies. We found that overall they were not confident in managing oncological emergencies and even less so in managing toxicities from more novel immunotherapy treatments. $70 \%$ of 134 responders felt an acute oncology guideline app would be useful. Between October 2017 and January 2018, 646 new users downloaded the app and there were 16,792 separate screen views. The app has now been downloaded in over 50 countries including the USA, India and France.

Authors: ${ }^{\text {A }}$ no affiliation provided; ${ }^{B}$ Wales Cancer Network, Wales; CVelindre Cancer Centre, Cardiff, UK

\section{Conclusion}

Acute cancer emergencies present to a wide range of healthcare professionals and ensuring widespread access to acute oncology guidelines for busy non-cancer specialists is challenging.

The development of a simple, easy to use, free to download app giving offline access to acute oncology guidelines aimed at non-specialist healthcare professionals has resulted in many downloads and user episodes suggesting that it is valued by staff. Multiple downloads outside Wales suggest that the format may be transferable to other geographical areas and specialties.

\section{Conflict of interest statement}

We have no conflict of interest to declare. 